\title{
OPEN Macrophages rely on extracellular serine to suppress aberrant cytokine production
}

\author{
Kento Kurita ${ }^{1,2,10}$, Hiroya Ohta ${ }^{1,3,9,10}$, Ibuki Shirakawa ${ }^{1,3,10}$, Miyako Tanaka ${ }^{1,3}$, \\ Yasuyuki Kitaura ${ }^{4}$, Yorihiro Iwasaki ${ }^{5}$, Takashi Matsuzaka ${ }^{6}$, Hitoshi Shimano ${ }^{6}$, Seiichiro Aoe ${ }^{7}$, \\ Hiroshi Arima ${ }^{2}$, Yoshihiro Ogawa ${ }^{1,8}$, Ayaka Ito ${ }^{1,3 \rrbracket}$ \& Takayoshi Suganami ${ }^{1,3 凶}$
}

A growing body of evidence indicates that cellular metabolism is involved in immune cell functions, including cytokine production. Serine is a nutritionally non-essential amino acid that can be generated by de novo synthesis and conversion from glycine. Serine contributes to various cellular responses, but the role in inflammatory responses remains poorly understood. Here, we show that macrophages rely on extracellular serine to suppress aberrant cytokine production. Depleting serine from the culture media reduced the cellular serine content in macrophages markedly, suggesting that macrophages depend largely on extracellular serine rather than cellular synthesis. Under serine deprivation, macrophages stimulated with lipopolysaccharide showed aberrant cytokine expression patterns, including a marked reduction of anti-inflammatory interleukin-10 expression and sustained expression of interleukine-6. Transcriptomic and metabolomics analyses revealed that serine deprivation causes mitochondrial dysfunction: reduction in the pyruvate content, the NADH/NAD+ ratio, the oxygen consumption rate, and the mitochondrial production of reactive oxygen species (ROS). We also found the role of mitochondrial ROS in appropriate cytokine production. Thus, our results indicate that cytokine production in macrophages is tightly regulated by the nutritional microenvironment.

Macrophages play multifunctional roles in immune responses, for which cytokine production is an essential process during infection and tissue injury. Cytokine production in macrophages is tightly regulated in response to the microenvironment at the site of inflammation, in order to avoid persistent inflammation and subsequent tissue damage ${ }^{1}$. In addition to a variety of pathogenic stimuli, an emerging field called "immunometabolism" proposes the involvement of cellular metabolism such as glycolysis and fatty acid oxidation in the regulation of immune cell function, including cytokine production ${ }^{2,3}$. In this context, we and others have previously demonstrated that saturated fatty acids induce proinflammatory cytokine expression in macrophages by activating signaling pathways downstream of toll-like receptor 4 (TLR4) and endoplasmic reticulum stress ${ }^{4-6}$. Our data also indicated that saturated fatty acids upregulate the expression of genes related to serine metabolism ${ }^{4}$, suggesting a complex link between the metabolic pathways under inflammatory conditions. Although recent studies have indicated the role of essential amino acids such as tryptophan and histidine in immune cell function ${ }^{7}$, the role of non-essential amino acids such as serine in macrophage cytokine production remains poorly understood.

Serine is considered a nutritionally non-essential amino acid, because it can be synthesized de novo from 3-phosphoglycerate, an intermediate in glycolysis, by phosphoglycerate dehydrogenase (PHGDH) ${ }^{8}$. Serine contributes to various cellular responses, such as nucleotide synthesis, methylation reactions, and antioxidant

\footnotetext{
${ }^{1}$ Department of Molecular Medicine and Metabolism, Research Institute of Environmental Medicine, Nagoya University, Nagoya, Aichi, Japan. ${ }^{2}$ Department of Endocrinology and Diabetes, Nagoya University Graduate School of Medicine, Nagoya, Aichi, Japan. ${ }^{3}$ Department of Immunometabolism, Nagoya University Graduate School of Medicine, Nagoya, Aichi, Japan. 'Laboratory Nutritional Biochemistry, Department of Applied Biosciences, Graduate School of Bioagricultural Sciences, Nagoya University, Nagoya, Aichi, Japan. ${ }^{5}$ Center for Diabetes and Endocrinology, The Tazuke Kofukai Medical Research Institute Kitano Hospital, Osaka, Osaka, Japan. ${ }^{6}$ Department of Endocrinology and Metabolism, Faculty of Medicine, University of Tsukuba, Tsukuba, Ibaraki, Japan. ${ }^{7}$ Department of Home Economics, Otsuma Women's University, Tokyo, Japan. ${ }^{8}$ Department of Medicine and Bioregulatory Science, Graduate School of Medical Sciences, Kyushu University, Fukuoka, Fukuoka, Japan. ${ }^{9}$ Department of Pharmacology, Faculty of Pharmaceutical Sciences, Tokushima Bunri University, Tokushima, Japan. ${ }^{10}$ These authors contributed equally: Kento Kurita, Hiroya Ohta and Ibuki Shirakawa. ${ }^{\square}$ email: aito@riem.nagoya-u.ac.jp; suganami@riem.nagoya-u.ac.jp
} 
a

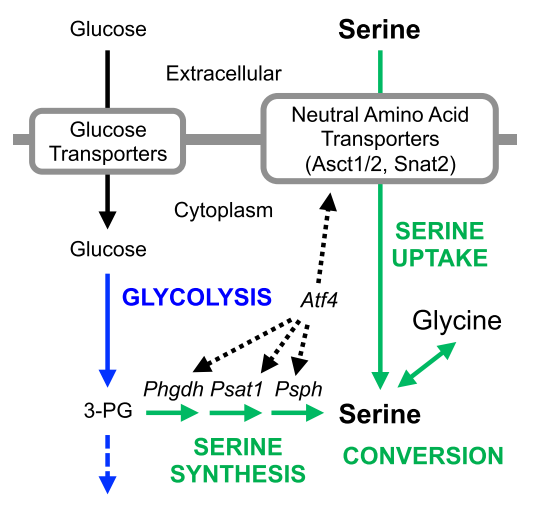

d

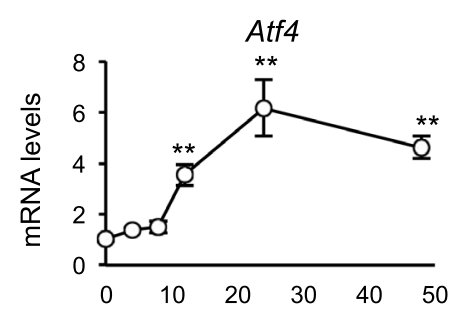

b

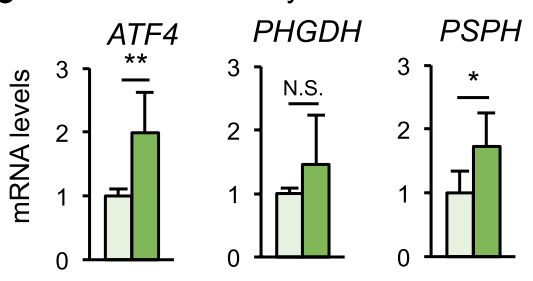

C

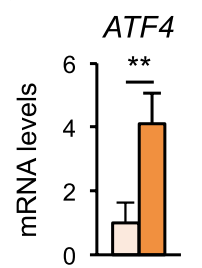

Healthy $\square$ Rheumatoid arthritis

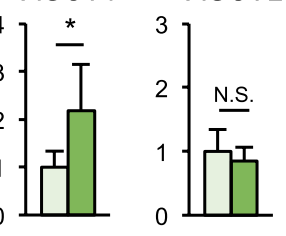

PHGDH PSPH ASCT1

ASCT2
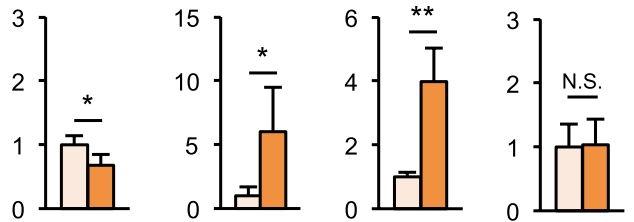

Phgdh
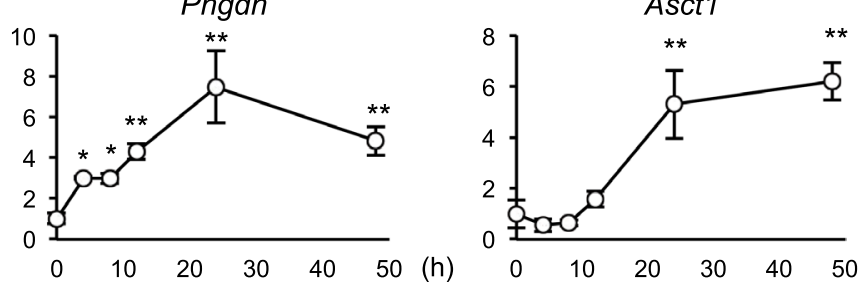

(h)

e
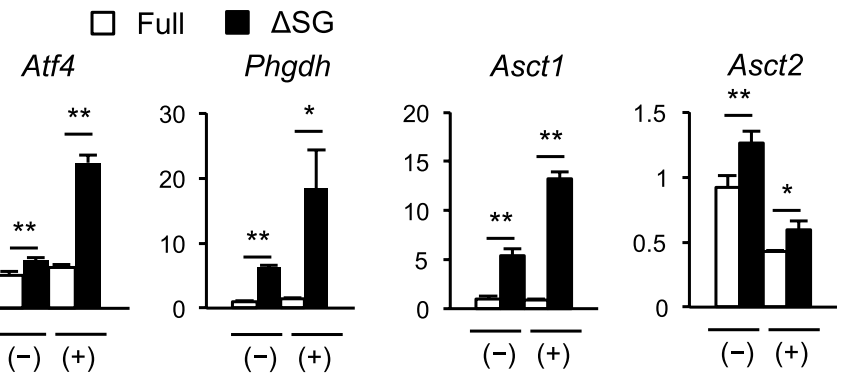

Snat2

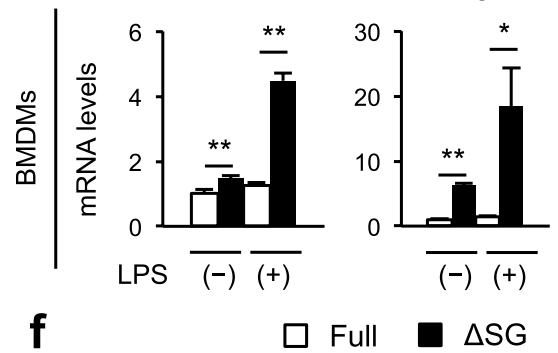

$$
\text { Atf4 Phgdh }
$$
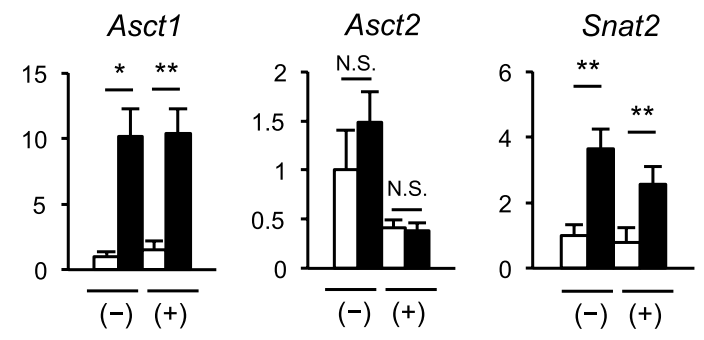

g
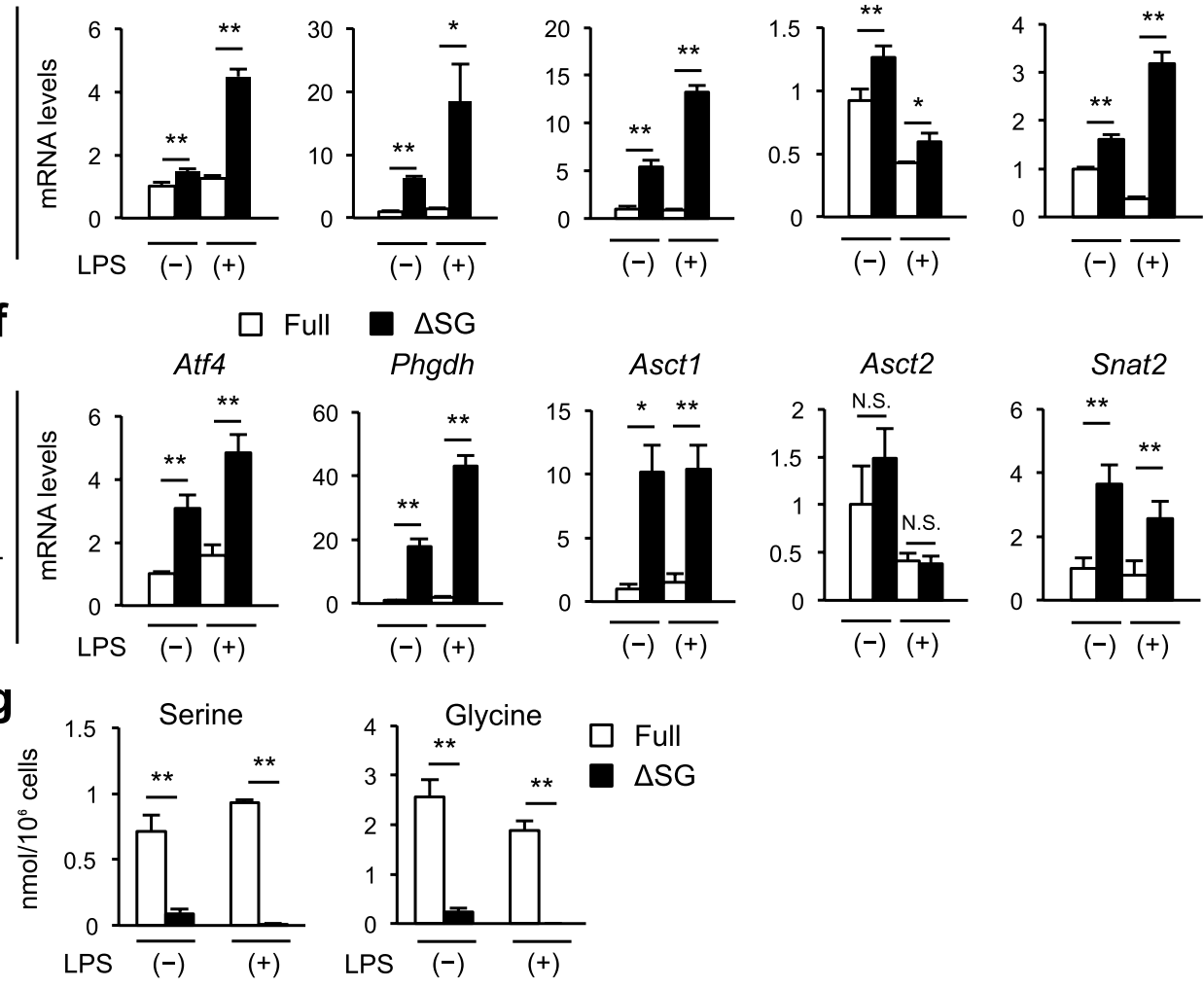

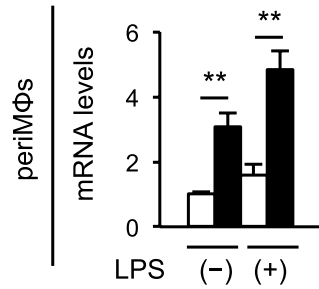

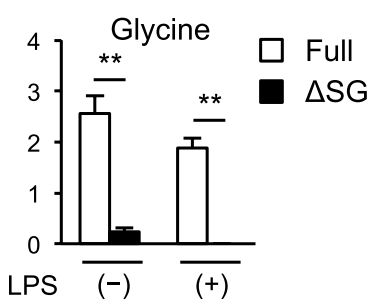


4Figure 1. Macrophages under inflammatory conditions require exogenous serine to maintain intracellular serine levels. (a) Schema of serine metabolism. Neutral amino acid transporters include ASCT1, ASCT2, and SNAT2. (b) Gene expression of transcription factor and enzymes for serine biosynthesis and neutral amino acid transporters in peripheral blood mononuclear cells (PBMC) of 10 patients infected with rotavirus compared to 8 age-matched healthy controls (GEO dataset: GSE2729). (c) Gene expression of transcription factor and enzymes for serine biosynthesis and neutral amino acid transporters in macrophages from synovial fluids of 5 patients with rheumatoid arthritis compared to PBMC of 3 healthy donors (GEO dataset: GSE10500). (d) Gene expression of Atf4, Phgdh and Asct1 in bone marrow-derived macrophages (BMDMs) at before and 4, 8, 12, 24, and $48 \mathrm{~h}$ after LPS stimulation. BMDMs were cultured in Full medium for $24 \mathrm{~h}$, followed by stimulation with LPS $(100 \mathrm{ng} / \mathrm{ml})$ for the indicated times $(\mathrm{n}=4)$. (e,f) Gene expression of Atf4, Phgdh, and neutral amino acid transporters in BMDMs (e) and peritoneal macrophages (periMФs) (f). BMDMs or periMФs were cultured in control (Full) or serine/glycine-depleted $(\triangle \mathrm{SG})$ medium for $24 \mathrm{~h}$, followed by stimulation with LPS (100 ng/ $\mathrm{ml}$ ) for $8 \mathrm{~h}(\mathrm{n}=3)$. (g) Cellular content of serine and glycine in periMФs analyzed by CE-TOF MS. PeriMФs were cultured in Full or $\Delta$ SG medium for $24 \mathrm{~h}$, followed by stimulation with LPS $(100 \mathrm{ng} / \mathrm{ml})$ for $6 \mathrm{~h}(\mathrm{n}=3)$. Values are means $\pm 95 \%$ CI. Statistical analysis was performed with unpaired $t$ test $(\mathbf{b}, \mathbf{c}, \mathbf{e}-\mathbf{g})$ and Dunnett's test (compared to $0 \mathrm{~h}(\mathbf{d})) .{ }^{\star} p<0.05 ;{ }^{* *} p<0.01$. See also Supplementary Figs. S1 and S2.

defense. Indeed, cellular serine is indispensable for cell proliferation in immune cells and tumors ${ }^{9-11}$. Cellular serine is also provided by uptake of extracellular serine via neutral amino acid transporters and by serine hydroxymethyltransferase (SHMT)-mediated conversion from glycine ${ }^{8}$. In this regard, certain cell types such as neurons and tumor cells cannot synthesize sufficient serine and thus are dependent on exogenous serine provided by surrounding supporting cells or dietary intake of serine ${ }^{12-14}$. Recently, several lines of evidence indicated that supplementation of serine in diet or injection of serine effectively attenuates proinflammatory cytokine expression in animal models of bacterial infection and tissue injury ${ }^{15-18}$. In stark contrast, serine deficiency is known to inhibit interleukin-1 $\beta$ (Il1 $\beta$ ) expression in macrophages through glutathione and one-carbon metabolism ${ }^{19,20}$. Thus, how serine regulates inflammatory responses remains controversial. It is also important to elucidate serine's mechanism of action, since cellular serine is derived from more than one source.

Here, we show that depleting serine from the culture media markedly reduces serine levels in macrophages, indicating that the cellular serine levels are not compensated by cellular synthesis or conversion from glycine. When deprived of serine, macrophages stimulated with lipopolysaccharide (LPS) exhibit aberrant cytokine expression patterns, among which expression of anti-inflammatory interleukin-10 (IL10) is markedly inhibited, thereby sustaining proinflammatory cytokine expression. A combination of transcriptomic and metabolomics analyses revealed that serine deprivation reduces cellular pyruvate content, its transport into mitochondria, and the $\mathrm{NADH} / \mathrm{NAD}^{+}$ratio. We also found that the mitochondrial dysfunction results in impaired production of reactive oxygen species (ROS) in mitochondria and causes aberrant cytokine expression. This study demonstrates that extracellular serine is required for LPS-induced IL10 production, thereby suppressing sustained proinflammatory cytokine expression.

\section{Results}

Macrophages under inflammatory conditions require exogenous serine to maintain intracellular serine levels. In most cell types, there are three major pathways to acquire serine, namely de novo serine synthesis, extracellular serine uptake, and conversion from glycine (Fig. $1 \mathrm{a})^{8,12}$. Because serine is considered to be produced mainly within each cell and the role of serine uptake is largely unknown, we first analyzed the expression of representative genes involved in serine metabolism, such as activating transcription factor 4 (ATF4, a transcription factor that promotes serine metabolism genes), phosphoglycerate dehydrogenase $(P H G D H$, a rate-limiting enzyme involved in serine biosynthesis), phosphoserine phosphatase ( $P S P H$, a key enzyme involved in serine biogenesis), and neutral amino acid transporters, including alanine serine cysteine transporters (ASCT1 and ASCT2), using publicly accessible gene expression omnibus (GEO) datasets. As a result, expression of ATF4, PSPH, and ASCT1 was upregulated in peripheral blood mononuclear cells of patients infected with rotavirus (Fig. $1 \mathrm{~b})^{21}$ and in synovial macrophages in patients with rheumatoid arthritis (Fig. 1c ${ }^{22}$ compared to their respective healthy controls. Overall, genes involved in serine metabolism were upregulated by lipopolysaccharide (LPS) stimulation in mouse bone marrow-derived macrophages (BMDMs) as well (Fig. 1d), whereas the gene expression pattern of PHGDH was distinct between the species (Fig. 1b-d), probably due to its complex regulatory mechanism ${ }^{23}$. These observations led us to speculate that the demand for serine increases in immune cells under inflammatory conditions.

We then cultured BMDMs and peritoneal macrophages (periMФs) in serine- and glycine-depleted medium ( $\triangle \mathrm{SG}$ medium) and stimulated with LPS. In this study, we used $\Delta$ SG medium so that serine would not be provided by the conversion of glycine to serine (Fig. 1a). The expression of Atf4, Phgdh and neutral amino acid transporters (Asct1, Asct2, and system A amino acid transporter 2 (Snat2)) was significantly increased in macrophages, when they were cultured in $\triangle S G$ medium compared with the control medium containing all amino acids (Full medium), and expression of some genes was further upregulated by LPS stimulation (Fig. 1e,f). Despite the increased expression of these genes, cellular serine and glycine were almost exhausted in periMФs (Fig. 1g, Supplementary Fig. S1) and BMDMs (Supplementary Fig. S2) cultured in $\Delta$ SG medium. These data indicate that deprivation of serine and glycine in culture media does not maintain their intracellular serine levels in macrophages during inflammation.

Serine and glycine deprivation results in aberrant cytokine expression in macrophages. Next, we determined the effect of serine and glycine deprivation on cytokine expression in cultured macrophages 

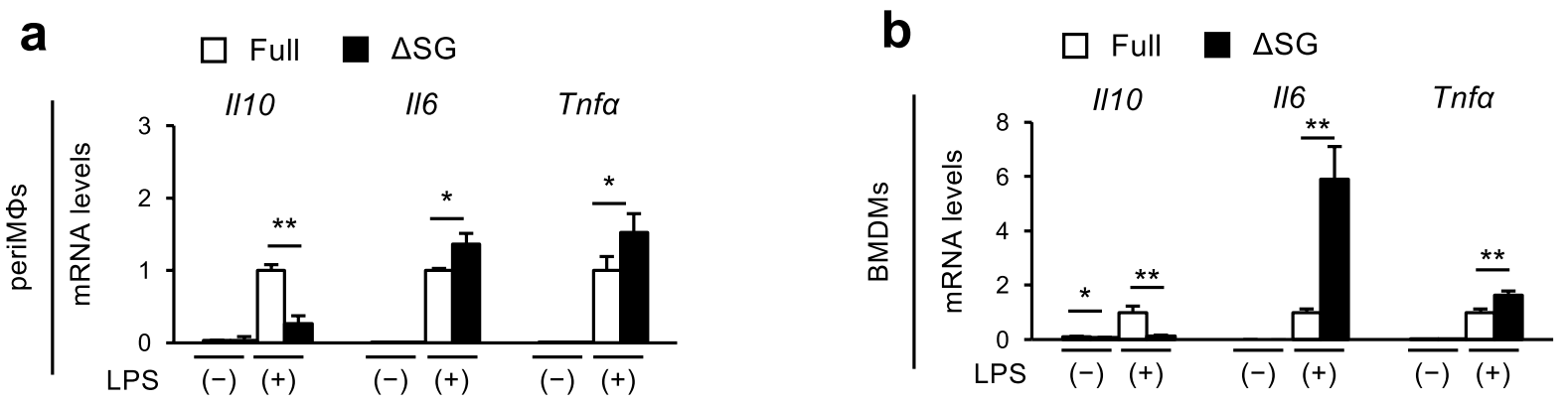

C

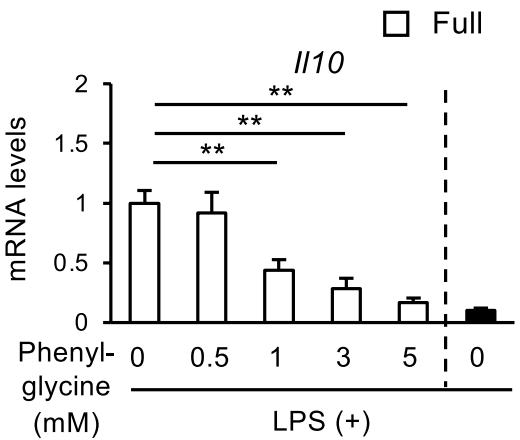

$\Delta S G$

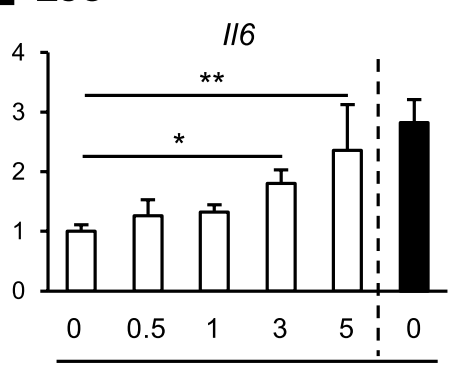

d $\quad \square$ siControl

siAsct1

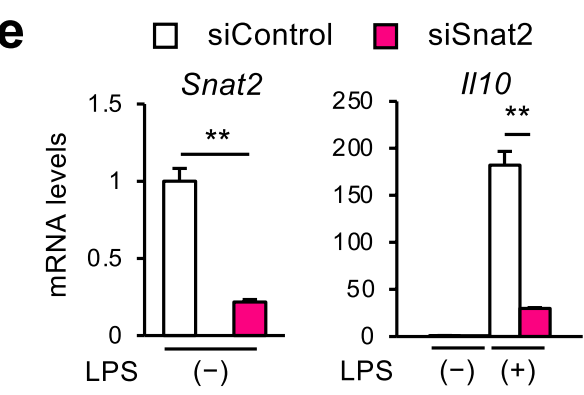

LPS (+)
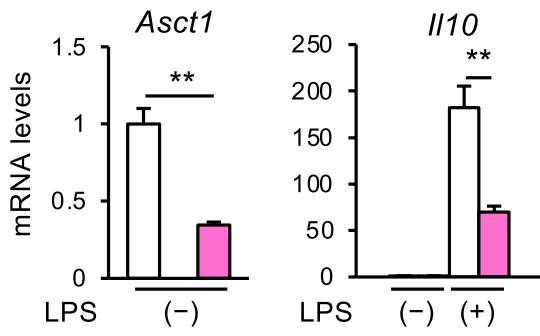

g
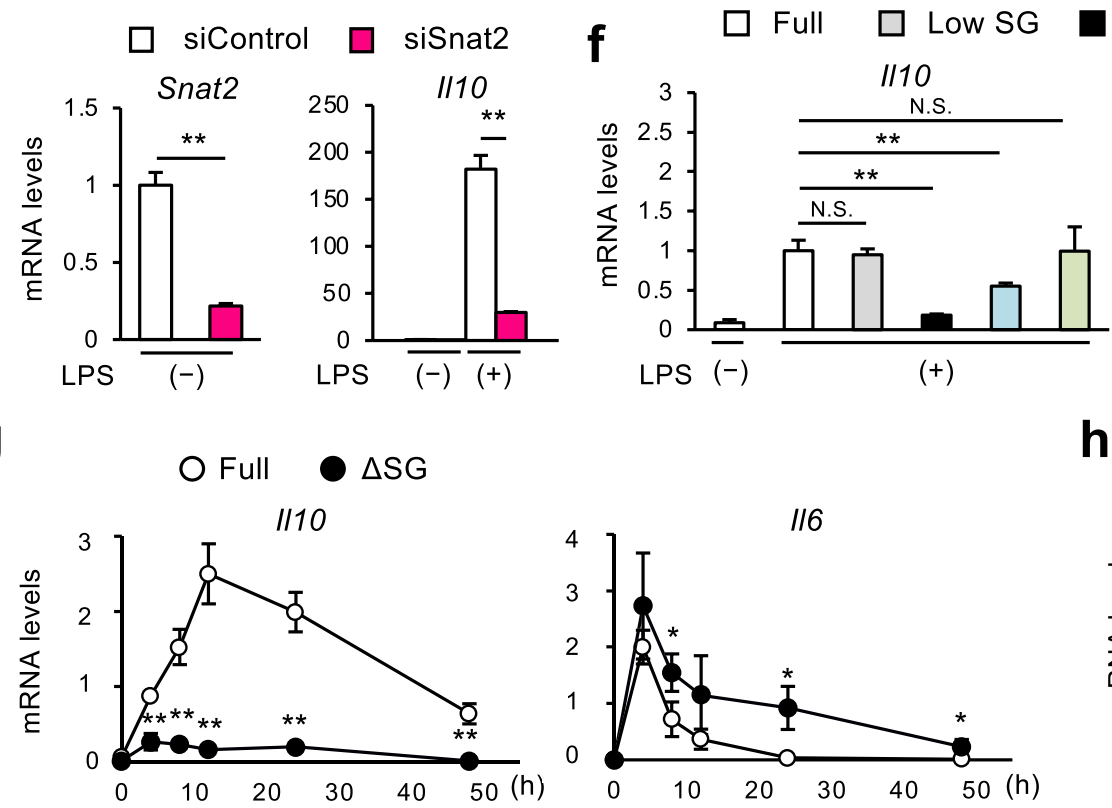

$\Delta S G \quad \square \Delta S$

$\Delta S \quad \square \Delta \mathrm{G}$

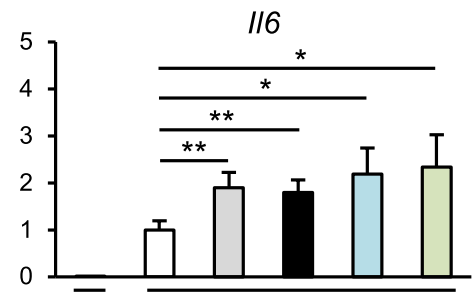

LPS (-)

$(+)$

h
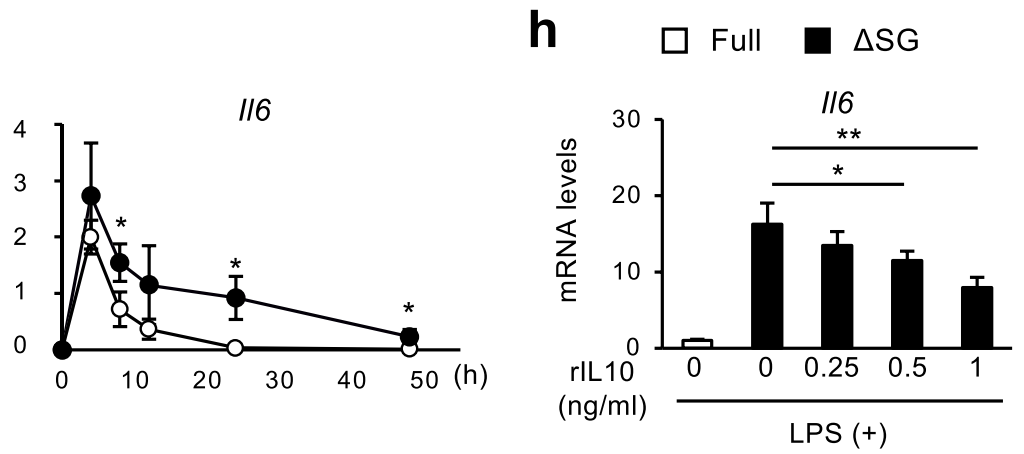

Figure 2. Serine and glycine deprivation results in aberrant cytokine expression in macrophages. (a,b) Gene expression of cytokines in periMФs (a) and BMDMs (b). PeriMФs and BMDMs were cultured in Full or $\triangle$ SG medium for $24 \mathrm{~h}$, followed by stimulation with LPS $(100 \mathrm{ng} / \mathrm{ml})$ for $8 \mathrm{~h}$ and $24 \mathrm{~h}$, respectively $(\mathrm{n}=3-4)$. (c) Gene expression of $I l 10$ and Il6 in BMDMs. BMDMs were cultured in Full or $\Delta$ SG medium with the indicated concentrations of 4-hydroxy-L-phenylglycine (an inhibitor of ASCT1 and ASCT2) for $24 \mathrm{~h}$, followed by stimulation with LPS (100 $\mathrm{ng} / \mathrm{ml})$ for $8 \mathrm{~h}(\mathrm{n}=4)$. (d,e) Gene expression of Asct1 and Il10 (E) and of Snat2 and Il10 (F) in J774 macrophages transfected with siAsct1 (e), siSnat2 (f), and siControl were stimulated with LPS (100 ng/ml) for $24 \mathrm{~h}(\mathrm{n}=4)$. (f) Gene expression of IllO and Il6 in BMDMs cultured in medium containing various levels of serine and glycine for 24 $\mathrm{h}$, followed by stimulation with LPS (100 ng/ml) for $8 \mathrm{~h}$. Full medium contains $400 \mu \mathrm{M}$ serine and $400 \mu \mathrm{M}$ glycine, Low SG medium contains $100 \mu \mathrm{M}$ serine and $100 \mu \mathrm{M}$ glycine, $\Delta \mathrm{SG}$ medium contains neither serine nor glycine, $\Delta \mathrm{S}$ medium contains no serine and $400 \mu \mathrm{M}$ glycine, and $\Delta \mathrm{G}$ medium contains $400 \mu \mathrm{M}$ serine and no glycine, $(\mathrm{n}=4)$. (g) Gene expression of $I l 10$ and Il6 in BMDMs at before and 4, 8, 12, 24, and $48 \mathrm{~h}$ after LPS stimulation. BMDMs were cultured in Full or $\Delta$ SG medium for $24 \mathrm{~h}$, followed by stimulation with LPS $(100 \mathrm{ng} / \mathrm{ml})$ for the indicated times $(\mathrm{n}=4)$. (h) Gene expression of Il6 in BMDMs. BMDMs were cultured in Full or $\Delta$ SG medium for $24 \mathrm{~h}$, treated with the indicated concentrations of recombinant IL10 (rIL10) for $4 \mathrm{~h}$, and then stimulated with LPS (100 ng/ml) for 24 $\mathrm{h}(\mathrm{n}=3)$. Values are means $\pm 95 \%$ CI. Statistical analysis was performed unpaired $t$ test $(\mathbf{a}, \mathbf{b}, \mathbf{d}-\mathbf{f})$ and Dunnett's test $(\mathbf{c}, \mathbf{g}, \mathbf{h})$ (compared to Full + phenylglycine $0 \mathrm{mM}(\mathbf{c}) ; \Delta \mathrm{SG}+\mathrm{rIL10} 0 \mathrm{ng} / \mathrm{ml}(\mathbf{g}) ;$ Full $+\mathrm{LPS}(+)(\mathbf{h})) .{ }^{*} p<0.05 ;{ }^{* *} p<0.01$. See also Supplementary Figs. S3 and S4. 
(Fig. 2, Supplementary Fig. S3). In both periMФs and BMDMs, treatment with LPS increased the expression of pro-inflammatory interleukin-6 (Il6) and tumor necrosis factor- $\alpha$ (Tnfa), together with anti-inflammatory Il10 in Full medium (Fig. 2a,b, white bars). Depleting serine and glycine from the medium markedly suppressed LPSinduced IL10 expression and production, while significantly augmenting LPS-induced expression and production of inflammatory cytokines and chemokines including IL6 and TNFa (Fig. 2a,b, Supplementary Fig. S3a-c). $\triangle$ SG medium did not affect the expression of these genes without LPS treatment (Fig. 2a,b). Similar results were observed when BMDMs were stimulated with lower doses of LPS (Supplementary Fig. S3d).

Next, we treated BMDMs cultured in Full medium with 4-hydroxy-L-phenylglycine (phenylglycine), an inhibitor of ASCT1, to block the incorporation of extracellular serine to BMDMs. Phenylglycine suppressed LPS-induced expression and production of IL10 while augmenting those of IL6, both in a dose-dependent manner (Fig. 2c, Supplementary Fig. S3e). Similar results were obtained using siRNA for Asct1 and Snat2 (Fig. 2d,e). Because the selectivity of Asct1 and Snat 2 for glycine is low, these findings suggest that extracellular serine is required to suppress LPS-induced aberrant cytokine production. To further distinct the role of serine and glycine, we cultured BMDMs in either medium containing lower concentrations (quarter amount of Full medium) of serine and glycine (Low SG medium), $\Delta$ SG medium, medium lacking only serine and not glycine ( $\Delta S$ medium), or medium lacking only glycine and not serine ( $\Delta \mathrm{G}$ medium). Similar to $\Delta S G$ medium, $\Delta S$ medium significantly suppressed LPS-induced Il10, while Low SG or $\Delta$ G medium did not (Fig. 2f), indicating that extracellular serine but not glycine is required for $I l 10$ expression. On the contrary, all these media including $\Delta \mathrm{G}$ medium significantly augmented LPS-induced Il6 (Fig. 2f), suggesting that both serine and glycine regulate Il6 expression. The distinct effects of glycine may be attributed to glutathione metabolism. As glycine is essential for glutathione synthesis (Supplementary Fig. S4a), the amount of glutathione was significantly reduced in periMФs cultured in $\Delta$ SG medium compared with Full medium (Supplementary Fig. S4b). Consistently, glutathione replenishment reversed only $I l 6$ expression ${ }^{24}$, whereas it did not affect $I l 10$ expression (Supplementary Fig. S4c).

In addition, time course analysis revealed that suppression of $I l 10$ by $\Delta$ SG was observed as early as $4 \mathrm{~h}$ after LPS stimulation, whereas increase of Il6 by $\Delta$ SG was evident only after $8 \mathrm{~h}$ and persisted up to $48 \mathrm{~h}$ (Fig. $2 \mathrm{~g}$ ). These observations led us to speculate that suppressed expression of $I l 10$ in macrophages cultured in $\Delta$ SG medium causes upregulation of Il6 expression at the later time points, as previous studies reported that IL10 is crucial for resolution of inflammation ${ }^{25}$. Notably, IL10 supplementation reversed the otherwise increased Il6 expression in $\Delta \mathrm{SG}$ medium in a dose-dependent manner (Fig. 2h). Collectively, these findings suggest that suppressed Il10 expression by deprivation of extracellular serine causes sustained inflammatory cytokine production. Thus, we sought to elucidate the molecular mechanism by which extracellular serine regulates $I l 10$ expression in cultured macrophages.

Deprivation of serine and glycine alters cellular metabolism in macrophages. To address the mechanism underlying Illo suppression by serine and glycine depletion, we performed transcriptomic and metabolomic profiling of LPS-stimulated periMФs cultured in $\triangle$ SG or Full medium (Fig. 3, Supplementary Table S1). Metabolomic analysis revealed that depletion of serine and glycine from the culture medium markedly reduced their intracellular levels, along with acetyl-CoA, malonyl-CoA, lactate, and pyruvate, whereas the amount of phosphoenolpyruvic acid (PEP) tended to increase. The gap between the amount of PEP and pyruvate between the media is consistent with a previous report that serine is an allosteric activator of PKM2 (the M2 subtype of pyruvate kinase $)^{26}$. The upregulation of genes related to de novo serine synthesis (Phgdh, phosphoserine aminotransferase 1 (Psat1), and $P s p h$ ) was verified by transcriptome analysis, and reflected reduced intracellular serine levels. Moreover, there was a decrease in the amount of malonyl-CoA, a substrate for lipogenesis, and in the expression of stearoyl-CoA desaturase-1 $(S c d 1)$, a key enzyme in fatty acid metabolism. Thus, serine and glycine deprivation reduced the levels of several metabolites in the glycolysis and lipogenesis pathways. On the other hand, there was no apparent change in the cellular content of metabolites related to the tricarboxylic acid (TCA) cycle (Fig. 3, Supplementary Table S1), which may be due to compensation by anaplerotic pathways for the TCA cycle or a reduced TCA cycle flux. Taken together, these observations led us to determine the effect of several metabolites on aberrant cytokine expression in macrophages cultured in $\triangle \mathrm{SG}$ medium. Replenishment of PEP, lactate, acetyl-CoA, and malonyl-CoA did not reverse the otherwise reduced expression of Il10 in $\Delta$ SG medium (Supplementary Fig. S5). Moreover, deficiency of sterol regulatory element-binding transcription factor 1 (Srebf1), a key enzyme of de novo lipogenesis, in BMDMs did not affect $I l 10$ and Il6 expression in $\Delta$ SG medium (Supplementary Fig. S6). Thus, we narrowed down the possible target molecules to just pyruvate.

Reduction of mitochondrial pyruvate transport by serine and glycine deprivation is a cause of aberrant cytokine production. Supplementation of pyruvate dose-dependently reversed the effect of serine and glycine deprivation on Il10 and Il6 expression in BMDMs (Fig. 4a). We verified these data by measuring of IL10 and IL6 concentrations in the media (Fig. 4b), suggesting the critical role of pyruvate in aberrant cytokine production in macrophages cultured in $\triangle S G$ medium. Pyruvate is imported into mitochondria via mitochondrial pyruvate carrier 1 (MPC1), and then oxidized to acetyl-CoA, which enters the TCA cycle (Fig. 3). Thus, we examined the effect of UK5099, an inhibitor of MPC1, on LPS-induced Il10 and Il6 expression in BMDMs cultured in Full medium. UK5099 treatment downregulated Il10 expression while upregulating Il6 expression, both in a dose-dependent manner (Fig. 4c). Although pyruvate replenishment and UK5099 treatment induced a modest increase in the concentrations of serine and glycine (Supplementary Fig. S7), it does not seem that these changes play a major role in regulating Il10 expression. Collectively, these findings indicate that mitochondrial pyruvate transport plays a key role in regulation of cytokine expression in cultured macrophages. 


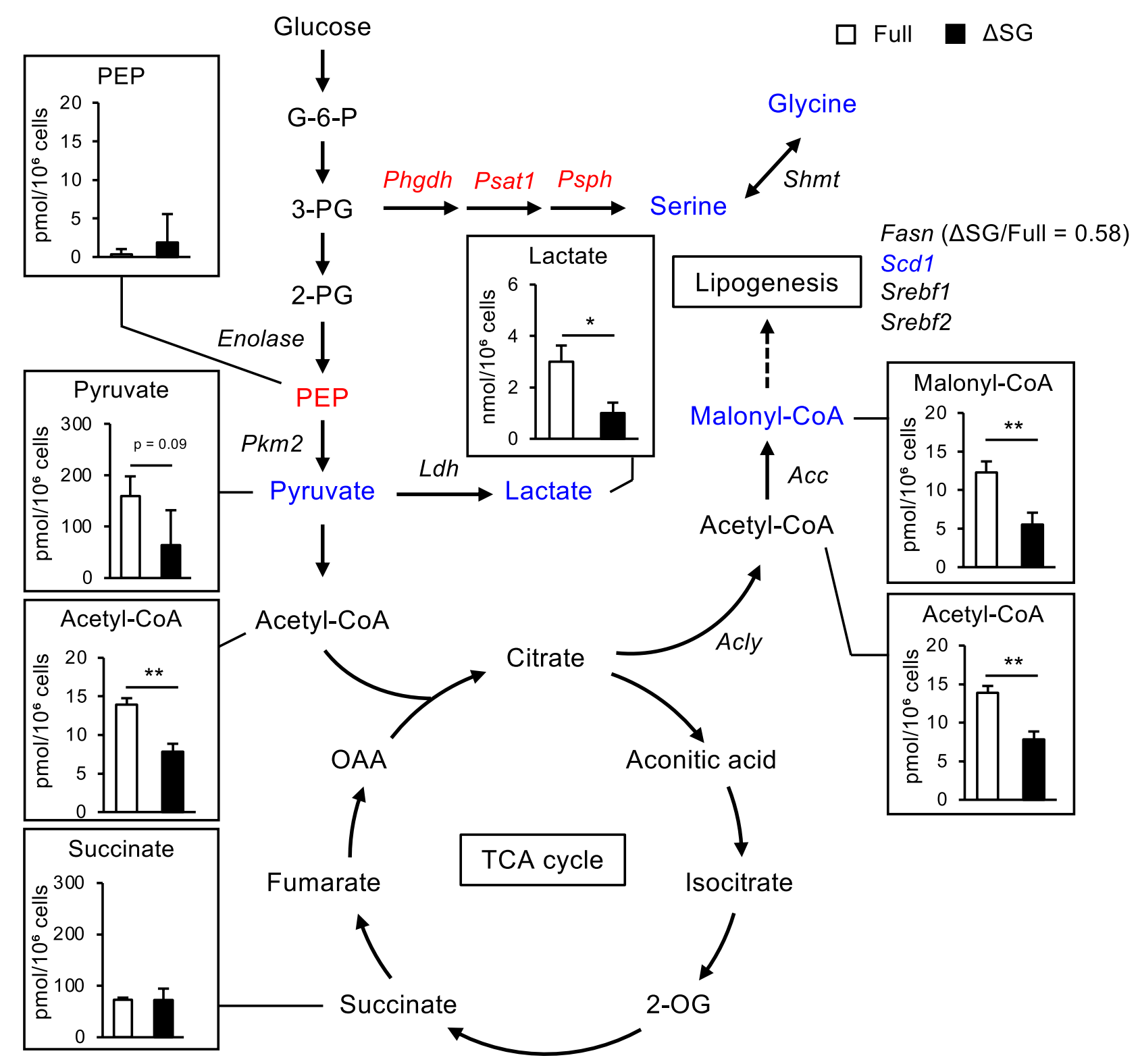

Figure 3. Serine and glycine deprivation alters cellular metabolism in macrophages. Transcriptomic and metabolic profiling in periMФs cultured in Full or $\Delta$ SG medium for $24 \mathrm{~h}$. Genes and metabolites whose expression and abundance were increased in $\Delta$ SG medium compared with Full medium $(\geq 2.0)$ are highlighted in red, and those whose expression and abundance were decreased in $\Delta \mathrm{SG}$ medium $(\leq 0.5)$ are highlighted in blue. Gene names are indicated in italics. Values are means $\pm 95 \%$ CI. $n=3$ and $n=4$ for metabolomic and transcriptomic analyses, respectively. Statistical analysis was performed with Student's $t$ test. ${ }^{\star} p<0.05 ;{ }^{*} p<0.01$. See also Supplementary Figs. S5 and S6.

Impaired production of mitochondrial ROS is involved in aberrant cytokine expression under serine and glycine-depleted conditions. Because mitochondria are multifunctional organelles, we sought to elucidate how serine and glycine deprivation influences mitochondrial functions in cultured macrophages. First, we visualized mitochondria using MitoTracker Red staining and found that $\Delta$ SG medium did not affect mitochondrial volume in BMDMs under both steady state and LPS-stimulated conditions (Fig. 5a,b). There was also no significant difference in expression of genes related to mitochondrial electron transport chain complexes between $\Delta$ SG and Full media (Fig. 5c). On the other hand, deprivation of serine and glycine significantly lowered the oxygen consumption rate (OCR) at steady state, and further lowered under LPS-stimulated conditions (Fig. 5d). These observations were recapitulated by UK5099 treatment in BMDMs cultured in Full medium (Fig. 5e). We also observed a significant reduction of the $\mathrm{NADH}$ content and the NADH/NAD ${ }^{+}$ratio in macrophages cultured in $\triangle S G$ medium relative to Full medium (Fig. 5f). Collectively, these data suggest that serine deprivation decreases pyruvate transport into mitochondria and $\mathrm{NADH}$ levels, which results in reduction of OXPHOS. 
a

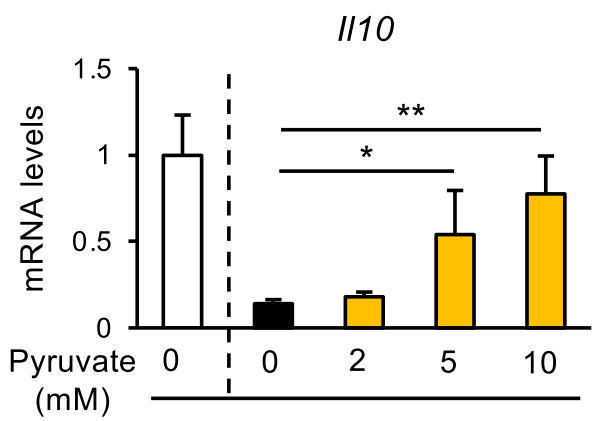

LPS (+)

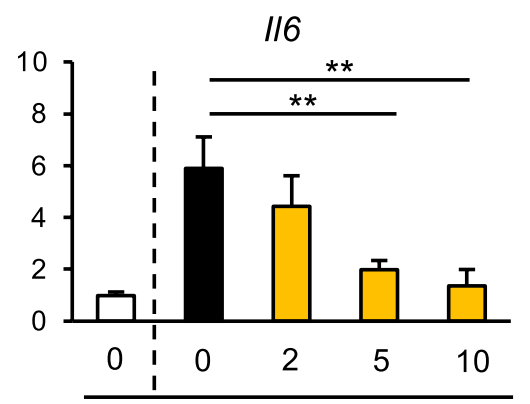

LPS (+)

b IL10

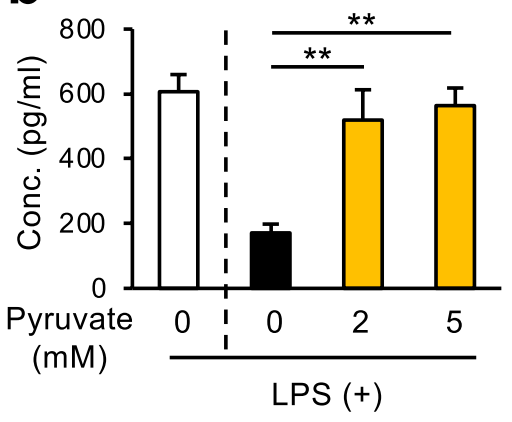

IL6

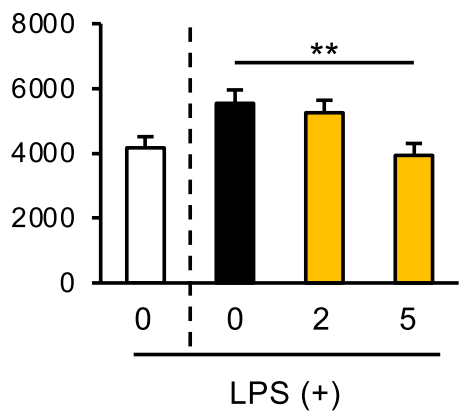

$\begin{array}{ll}\square & \text { Full } \\ \square & \Delta S G+\text { Vehicle } \\ \square & \Delta S G+\text { Pyruvate }\end{array}$

116
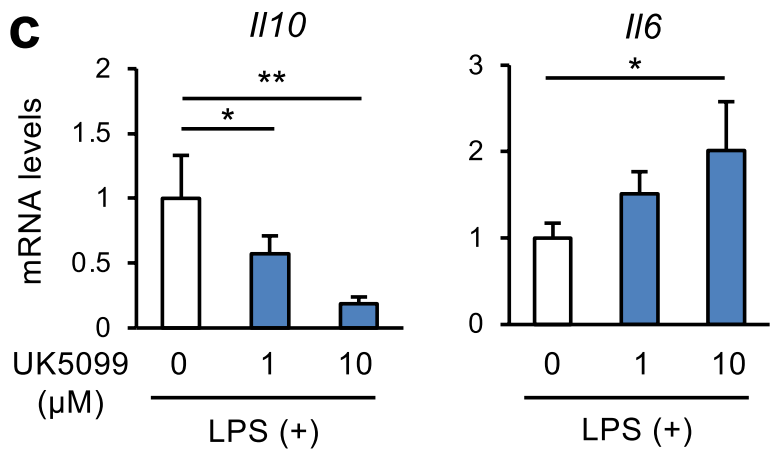

Full + Vehicle

Full + UK5099

Figure 4. Reduction of mitochondrial pyruvate transport by serine and glycine deprivation is a cause of aberrant cytokine production. $(\mathbf{a}, \mathbf{b})$ Gene expression (a) and production (b) of cytokines in BMDMs. BMDMs were cultured in Full or $\Delta S G$ medium with indicated concentration of pyruvate for $24 \mathrm{~h}$, followed by stimulation with LPS (100 ng/ml) for $24 \mathrm{~h}(\mathrm{n}=4)$. (c) Gene expression of Il10 and Il6 in BMDMs. BMDMs were cultured in Full with indicated concentration of UK5099 (an inhibitor of mitochondrial pyruvate carrier) for $24 \mathrm{~h}$, followed by stimulation with LPS $(100 \mathrm{ng} / \mathrm{ml})$ for $24 \mathrm{~h}(\mathrm{n}=4)$. Values are means $\pm 95 \% \mathrm{CI}$. Statistical analysis was performed with one-way ANOVA Dunnett's test (compared to $\Delta \mathrm{SG}+$ Vehicle $(\mathbf{a}, \mathbf{b})$; Full + Vehicle $(\mathbf{c})) .{ }^{\star} p<0.05$; ${ }^{* *} p<0.01$. See also Supplementary Figs. S5, S6, and S7.

Mitochondria is one of major sources of ROS. Although mitochondrial ROS (mROS) has been recognized as by-products of the TCA cycle and electron transport chain, recent evidence points to the role of mROS in various biological functions, including cytokine expression ${ }^{27}$. Using MitoSOX, a mitochondrial superoxide indicator, we found that LPS-induced mROS was significantly reduced in BMDMs cultured in $\triangle$ SG medium compared to Full medium, whereas there was no difference at steady state between the media (Fig. 6a). Blocking mROS using MitoTEMPO, a mitochondria-targeted antioxidant, effectively suppressed LPS-induced Il10 expression while augmenting that of $I l 6$ (Fig. 6b). These data were confirmed using other chemicals suppressing mROS production through complex I and III (S1QEL and S3QEL, respectively) (Fig. 6c). Furthermore, MitoTEMPO treatment almost abolished the effect of pyruvate supplementation on Il10 and Il6 expression in $\Delta$ SG medium (Fig. 6d). In contrast, a specific NADPH-oxidase inhibitor apocynin to block ROS production mainly on plasma membrane but not in mitochondria, did not affect LPS-induced IllO and Il6 expression in Full medium, suggesting that ROS differentially regulates cytokine expression depending on where it is produced (Fig. 6e). Collectively, these findings suggest that serine and glycine deprivation induces aberrant cytokine expression, at least partly, through impaired production of mROS in cultured macrophages (Supplementary Fig. S8). 
a

MitoTracker/

Hoechst

b
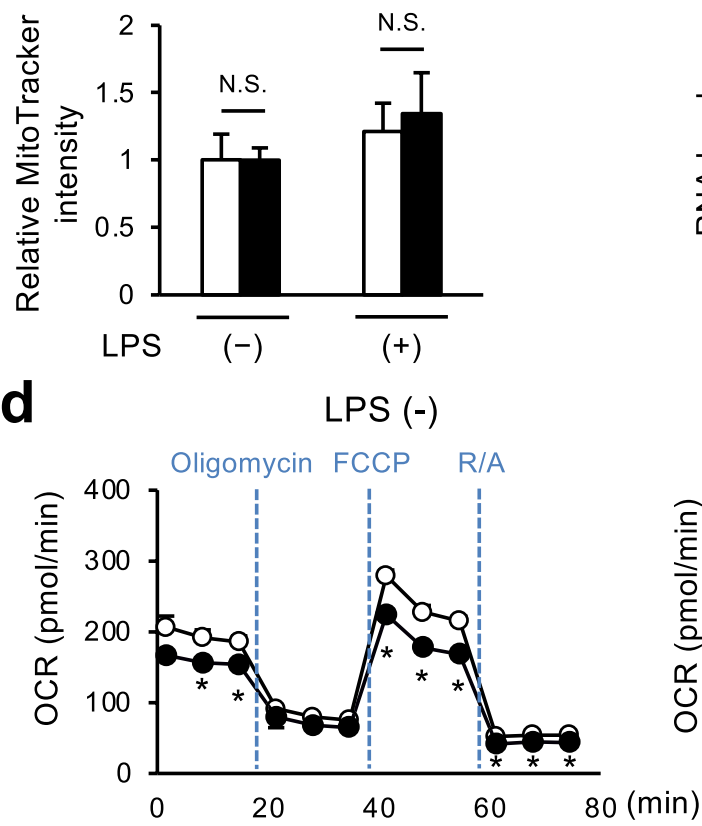

LPS (-)

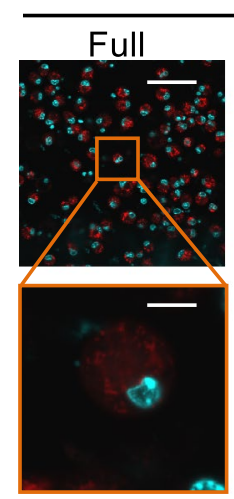

Full

$\Delta S G$

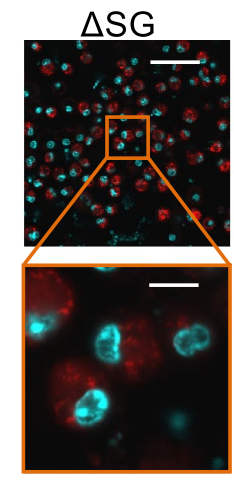

C

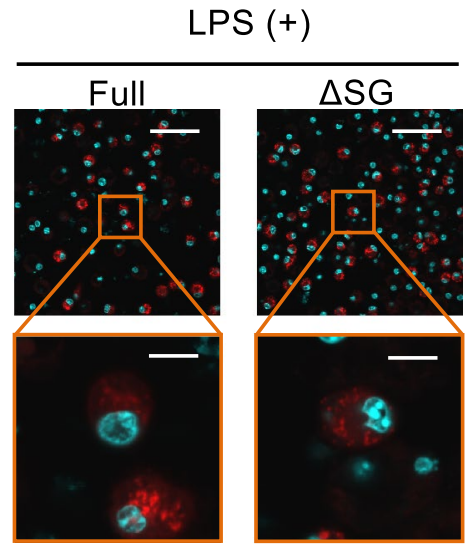

$\Delta S G$

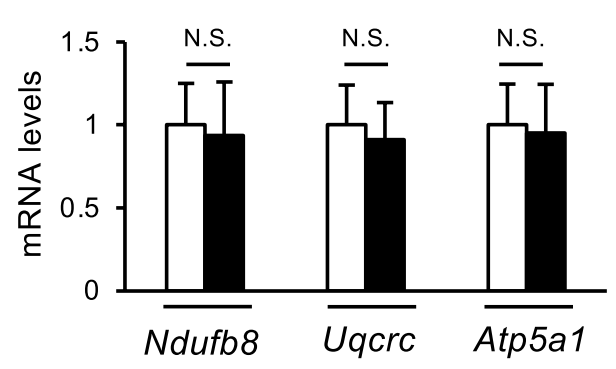

LPS (+)

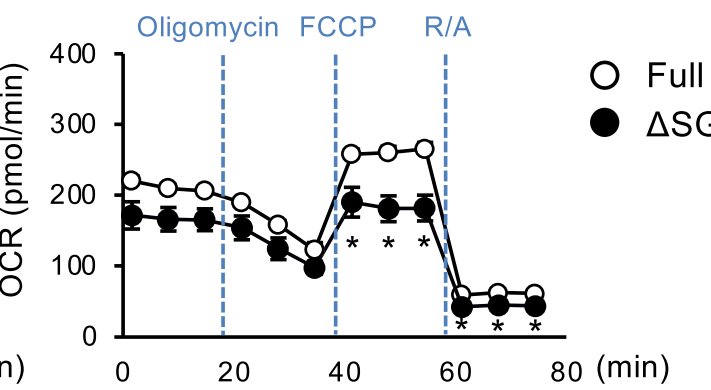

e $\bigcirc$ Full + Vehicle $\Delta$ Full + UK5099

\section{f}

Full

$\Delta S G$

LPS (+)
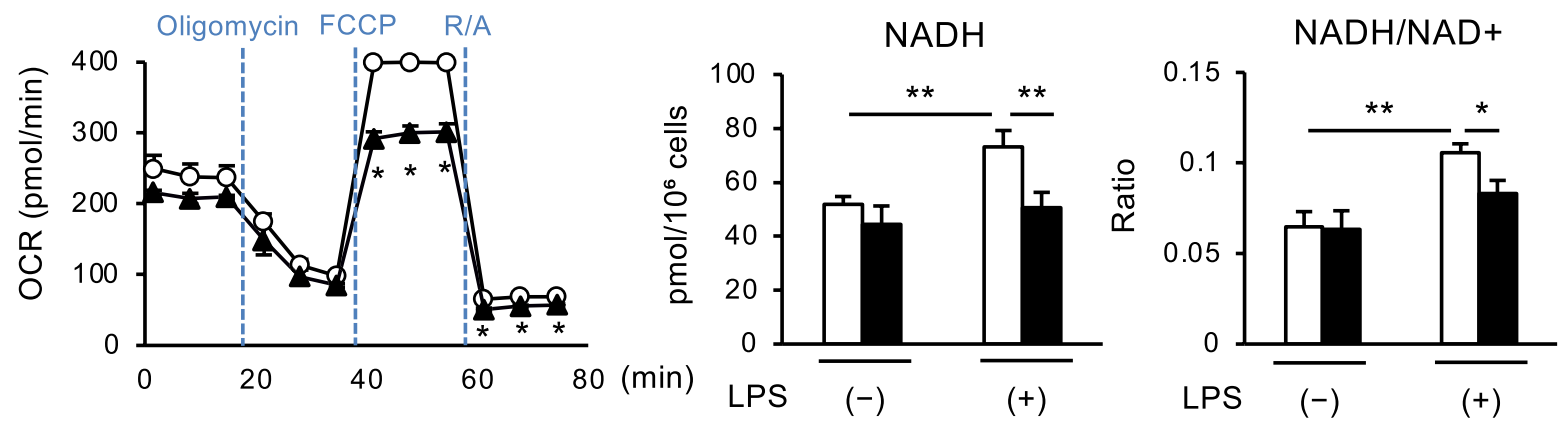
४Figure 5. Deprivation of serine and glycine results in mitochondrial dysfunction in macrophages. (a, b) Representative images of mitochondria visualized by MitoTracker Red staining in BMDMs. Nuclei were counterstained with Hoechst33342. Scale bars, $50 \mu \mathrm{m}$ (upper) and $300 \mu \mathrm{m}$ (lower) (a). Flow cytometry analysis of mitochondrial mass in BMDMs by MitoTracker Red staining (b). BMDMs were cultured in Full or $\triangle$ SG medium for $24 \mathrm{~h}$, followed by stimulation with LPS $(100 \mathrm{ng} / \mathrm{ml})$ for $8 \mathrm{~h}(\mathrm{n}=4)$. (c) Gene expression of mitochondrial OXPHOS complexes in BMDMs. Ndufb8, Uqcrc and Atp5a1 are the genes of complex I, III and $\mathrm{V}$, respectively. BMDMs were cultured in Full or $\Delta \mathrm{SG}$ medium for $24 \mathrm{~h}(\mathrm{n}=3)$. (d) Oxygen consumption rate (OCR) of BMDMs. BMDMs were cultured in Full or $\triangle \mathrm{SG}$ medium for $24 \mathrm{~h}$ followed by stimulation with vehicle (left) or LPS (100 ng/ml, right) for $6 \mathrm{~h}(\mathrm{n}=3)$. R/A: rotenone and antimycin. (e) OCR of BMDMs. BMDMs were cultured in Full or $\Delta$ SG medium with UK5099 $(1 \mu \mathrm{M})$ for $24 \mathrm{~h}$, followed by stimulation with LPS $(100 \mathrm{ng} / \mathrm{ml})$ for $6 \mathrm{~h}(\mathrm{n}=3)$. (f) Cellular content of NADH and NADH/NAD + in periMФs. PeriMథs were cultured in Full or $\Delta$ SG medium for $24 \mathrm{~h}$, followed by stimulation with LPS $(100 \mathrm{ng} / \mathrm{ml})$ for $6 \mathrm{~h}(\mathrm{n}=3)$. Values are means $\pm 95 \%$ CI. Statistical analysis was performed with unpaired $t$ test $(\mathbf{b}-\mathbf{e})$ with Bonferroni correction within treatments $(0-20,20-40,40-60,60-80 \mathrm{~min})(\mathbf{d}, \mathbf{e})$ and Dunnett's test (f) (compared to Full + LPS). ${ }^{*} p<0.05 ;{ }^{* *} p<0.01 ; N S$ not significant.

\section{Discussion}

Because inflammatory responses such as cytokine production, chemotaxis, and phagocytosis are energetically demanding processes, immune cells activate cellular metabolism to generate fuel under inflammatory conditions $^{28}$. In this study, we have provided evidence that macrophages largely rely on exogenous serine to maintain the cellular serine levels during inflammation. This phenotype resembles that of neurons, which do not express key enzymes involved in de novo serine synthesis. Hence, surrounding glial cells supply serine to neurons via ASCT $1^{14}$. However, unlike neurons, macrophages under serine deprivation upregulate the expression of genes related to serine metabolism. Why do macrophages require exogenous serine? It is probably attributed to the increased demand of serine under inflammatory conditions. Indeed, this study revealed that LPS stimulation induces the expression of genes related to de novo serine synthesis and neutral amino acid transporters in macrophages cultured in the Full medium. Consistently, monocytic cells of patients with a certain infectious disease or autoimmune disease exhibit similar expression patterns ${ }^{21,22}$, suggesting that de novo synthesis does not meet the high demand of serine. Accordingly, this study uncovered a fundamental mechanism, by which macrophages adapt to the nutritional microenvironment at the site of inflammation and modulate inflammatory cytokine production.

In this study, we used a combination of metabolomic and transcriptomic analyses to show that pyruvate, among a variety of other metabolites, is responsible for extracellular serine-regulated cytokine expression in macrophages. This notion is supported by several lines of evidence. For instance, Márquez et al. ${ }^{29}$ reported that inhibiting pyruvate transport to mitochondria by UK5099 suppresses IL10 expression in bone marrow-derived dendritic cells. Palsson-McDermott et al. ${ }^{30}$ showed that the activation of PKM2 or increased cellular pyruvate levels induce the expression of IL10 while suppressing IL1 $\beta$ in bone marrow-derived macrophages. Since serine is required for allosteric activation of PKM2, it is reasonable that cellular pyruvate levels are reduced under serinedepleted conditions ${ }^{26}$. In addition, serine racemase is supposed to produce pyruvate from serine $\mathrm{e}^{31,32}$. Indeed, our transcriptomic data show that peritoneal macrophages express serine racemase at higher levels under serinedepleted conditions than at steady state (Kurita et al., unpublished observations), which may be a compensatory mechanism for replenishing cellular pyruvate. Thus, pyruvate is a key metabolite for regulating inflammatory responses in immune cells, apart from its essential role in cellular energy metabolism.

Next, we must discuss the critical role of serine in one-carbon metabolism. Serine acts as a one-carbon donor, contributing to nucleotide synthesis, methylation reactions, and antioxidant production ${ }^{8}$. Substantial evidence has already shown that various cancer cells are dependent on serine in terms of cell proliferation and antioxidant defense, providing a novel therapeutic target for treating cancers ${ }^{33,34}$. Similarly, serine is required for T cell expansion in acquired immune respons $\mathrm{e}^{10}$. On the other hand, macrophages proliferate only under limited conditions in vivo, and little is known about the role of serine in non-proliferating cells. In this regard, several studies have reported that excessive serine administration can suppress inflammatory responses in certain animal models, by increasing glutathione synthesis ${ }^{15-19}$. Interestingly, our data indicate that glutathione replenishment does not affect Il10 expression in macrophages, whereas we confirmed that glutathione supplementation effectively suppresses Il6 expression. Nevertheless, we do not exclude the involvement of one-carbon metabolism in extracellular serine-regulated $I l 10$ expression, because Yu et al. ${ }^{20}$ recently reported that serine supports IL1 $\beta$ expression through S-adenosylmethionine production and histone methylation.

Amongst the myriads of cytokines, IL10 plays a critical role in resolving inflammation ${ }^{25}$. Macrophages are one of the major cellular sources of IL10 production, and also the main target cell type of the anti-inflammatory effects of IL10. Considerable attention has hitherto been paid to the pathophysiologic role of IL10 in various diseases. In terms of the mechanism of action, IL10 opposes the switch to the metabolic program induced by inflammatory stimuli ${ }^{35}$. However, the transcriptional regulation of IL10 remains incompletely understood. Using ROS inhibitors specific for mitochondria and NADPH oxidase, this study provides evidence that ROS production in mitochondria but not by NADPH oxidase is required for maximal expression of IL10 in LPS-treated macrophages. Consistently, mROS skews macrophage polarization toward an anti-inflammatory M2 phenotype ${ }^{36}$. Mills et al. ${ }^{37}$, however, have reported that mROS suppresses IL10 expression in macrophages. Therefore, further studies are needed to clarify the context-dependent effects of mROS on IL10 expression and the molecular mechanisms downstream of mROS. 

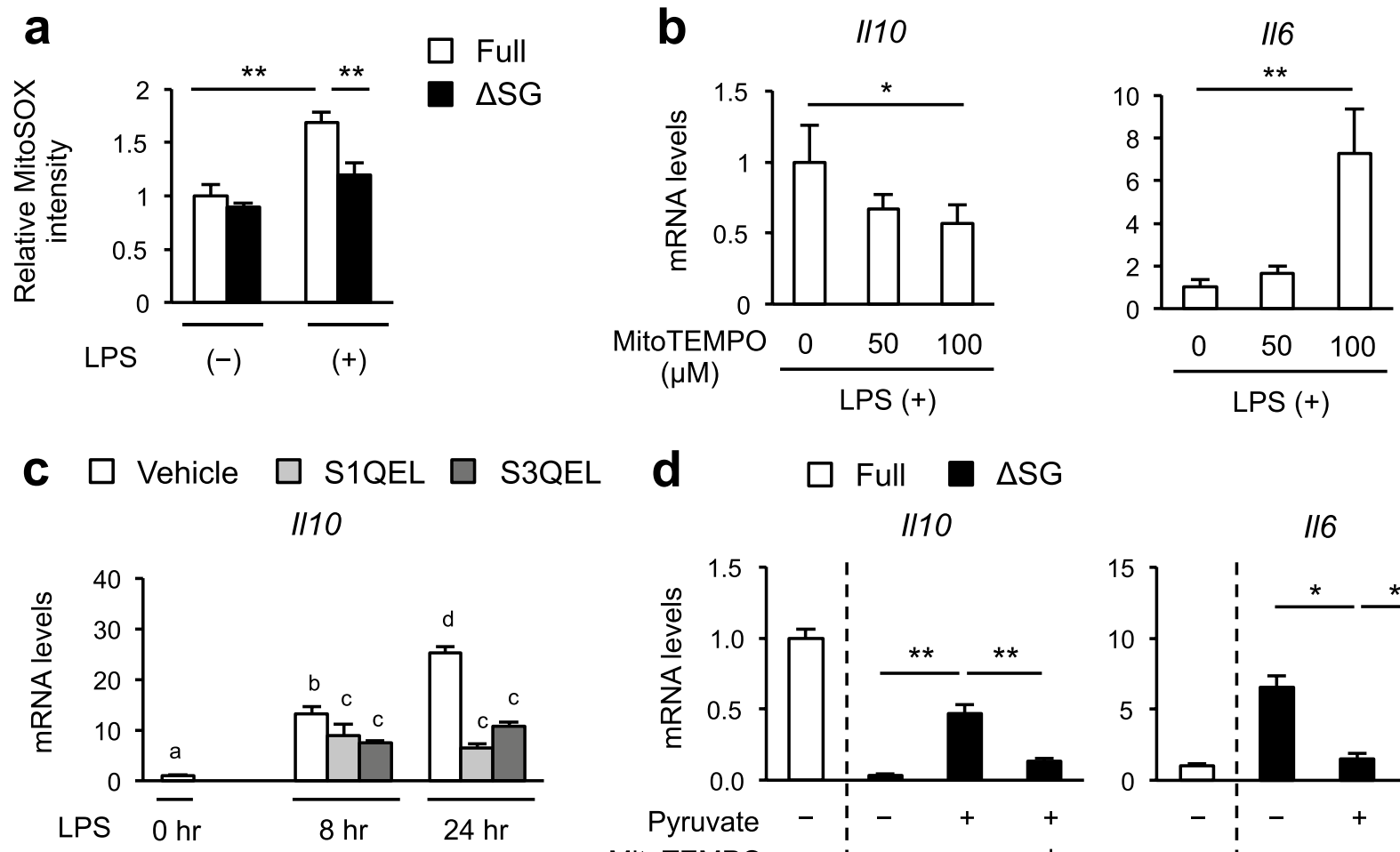

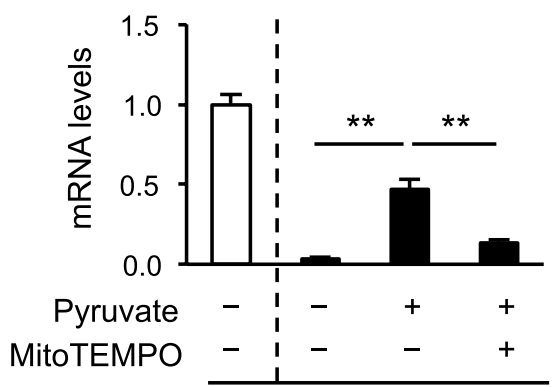

LPS (+)

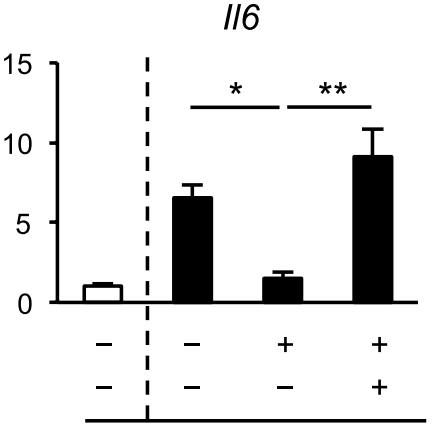

LPS (+)

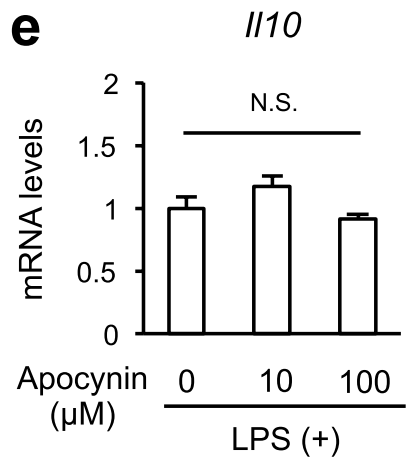

$1 / 6$

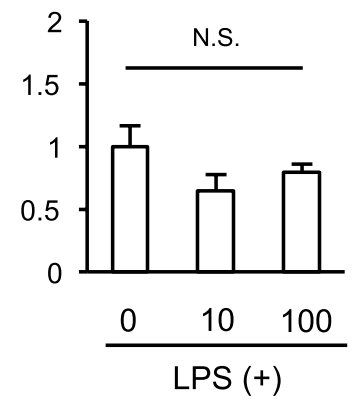

Figure 6. Impaired production of mitochondrial ROS by serine and glycine deprivation is a cause of aberrant cytokine expression. (a) Flow cytometry analysis of mitochondrial ROS production in BMDMs using MitoSOX (a mitochondrial superoxide indicator). BMDMs were cultured in Full or $\Delta \mathrm{SG}$ medium for $24 \mathrm{~h}$, followed by stimulation with LPS $(100 \mathrm{ng} / \mathrm{ml})$ for $8 \mathrm{~h}(\mathrm{n}=4)$. (b) Gene expression of $I l 10$ and $I l 6$ in BMDMs. BMDMs were cultured in Full medium with the indicated concentrations of MitoTEMPO (a mitochondria-targeted superoxide dismutase) for $24 \mathrm{~h}$, followed by stimulation with LPS $(100 \mathrm{ng} / \mathrm{ml})$ for $24 \mathrm{~h}(\mathrm{n}=4)$. (c) Gene expression of Il10 in BMDMs. BMDMs were cultured in Full medium for $24 \mathrm{~h}$, treated with S1QEL or S3QEL (suppressors of complex 1 and 3, respectively) for $24 \mathrm{~h}$, and then stimulated with LPS (100 ng/ml) for $8 \mathrm{~h}$ or $24 \mathrm{~h}(\mathrm{n}=4)$. (d) Gene expression of Il10 and Il6 in BMDMs. BMDMs were cultured in Full or $\Delta$ SG medium with pyruvate $(10 \mathrm{mM})$ and/or MitoTEMPO $(100 \mu \mathrm{M})$ for $24 \mathrm{~h}$, followed by stimulation with LPS $(100 \mathrm{ng} / \mathrm{ml})$ for $24 \mathrm{~h}(\mathrm{n}=4)$. (e) Gene expression of Il10 and Il6 in BMDMs. BMDMs were cultured in Full medium for 24 $\mathrm{h}$, treated with the indicated concentrations of apocynin for $1 \mathrm{~h}$, then stimulated with LPS (100 $\mathrm{ng} / \mathrm{ml})$ for $8 \mathrm{~h}$ $(n=4)$. Values are means $\pm 95 \%$ CI. Statistical analysis was performed with Dunnett's test $(\mathbf{a}, \mathbf{b}, \mathbf{d}, \mathbf{e})$ (compared to Full + LPS (a); Full + MitoTEMPO $0 \mu \mathrm{M}(\mathbf{b}) ; \Delta \mathrm{SG}+$ pyruvate + MitoTEMPO $0 \mu \mathrm{M}(\mathbf{d}))$ and Tukey-Kramer's test (c) (suffixed superscript letters differ significantly, $p<0.05$ ). ${ }^{\star} p<0.05$; ${ }^{*} p<0.01$; NS not significant.

In summary, we have demonstrated that macrophages require exogenous serine under inflammatory conditions to maintain their intracellular serine levels, in spite of increased expression of genes related to de novo serine synthesis and neutral amino acid transporters. Serine deprivation resulted in aberrant cytokine expression, particularly marked suppression of Il10 expression, in macrophages. Our data also showed that the reduced pyruvate amount and its transport to mitochondria under serine deprivation suppress mROS, thereby inhibiting Il10 expression. With regard to the clinical implications, similar metabolic changes may be observed in monocytic cells of patients with either viral infection or autoimmune disease $\mathrm{e}^{21,22}$. Since the dose of LPS mainly used 
in this study is above the physiological levels, future studies are required to elucidate the metabolic changes that macrophages undergo in response to various inflammatory conditions in vivo. This study provides insight into novel molecular mechanisms regulating cytokine expression in macrophages, in response to the surrounding nutritional conditions.

\section{Methods}

All methods were carried out in accordance with the ARRIVE guidelines.

Resources and primers for Q-PCR. Information of key resources and primers for Q-PCR used in this study are shown in Supplementary Tables S2 and S3.

Animals and cell culture. C57BL/6J mice were purchased from CLEA Japan or Japan SLC. All animals were housed in a temperature-, humidity- and light-controlled animal room (12 h light and $12 \mathrm{~h}$ dark cycle), and allowed free access to water and food (CE-2, CLEA Japan). Primary peritoneal macrophages (periMФs) were obtained from 3\% thioglycollate-treated mice 4 days after the injection. Murine bone marrow-derived macrophages (BMDMs) were obtained as described previously ${ }^{38}$. Briefly, bone marrow cells were isolated from femurs and tibias and differentiated in Iscove's Modified Dulbecco's Medium (Gibco) supplemented with 50 $\mathrm{ng} / \mathrm{mL}$ recombinant human macrophage colony stimulating factor (M-CSF) (PeproTech) and 20\% fetal bovine serum (FBS) for 6 days. J774 macrophages were cultured in Dulbecco's Modified Eagle Medium (DMEM) (Nacalai Tesque) with $10 \%$ FBS.

Gene expression and microarray analysis. Quantitative real-time PCR was performed as described ${ }^{4}$. In brief, total RNA was extracted from cells using Sepasol (Nacalai Tesque) and $10 \mathrm{ng}$ of cDNA was used for realtime PCR amplification using SYBR Green Master mix (Thermo Fisher Scientific) and StepOne Plus instrument (Applied Biosystems). The primers used in this study are listed in Supplementary Table S3. Data were normalized to 36B4 and relative gene expression was calculated using comparative Ct method. For microarray analysis, RNA was purified using RNeasy MinElute Cleanup Kit, pooled from $n=4$ biological replicates and processed at National Cerebral and Cardiovascular Center Hospital (Osaka, Japan) using Affymetrix GeneChip Mouse Genome 430 2.0 Arrays. Data was analyzed using Genespring GX (Agilent) and have been deposited in NCBI's Gene Expression Omnibus (GSE156325, https://www.ncbi.nlm.nih.gov/geo/query/acc.cgi?acc=GSE156325).

siRNA transfection. J774 macrophages were transfected with $50 \mathrm{nM}$ siRNA targeted control GFP-22 (1022064, QIAGEN), Asct1 (MSS225811, Thermo Fisher) or Snat2 (MSS289807, Thermo Fisher) using Lipofectamine RNAiMAX (Thermo Fisher Scientific) according to the manufacturer's instructions. Cells were incubated for $24 \mathrm{~h}$ prior to the assays. Knockdown of target genes was validated by real-time PCR.

Cytokine production measurements. Cell-free supernatants were collected and the concentration of IL10 and IL6 in culture medium were measured by ELISA kits (R\&D) according to manufacturer's protocols.

Oxygen consumption rate assays. Real-time oxygen consumption was measured using XFp Extracellular Flux analyzer (Agilent Technologies). BMDMs were seeded into Agilent Seahorse XFp Cell Culture Miniplate at a density of $1.0 \times 10^{5}$ cells/well in Full or $\Delta$ SG medium and cultured overnight. Prior to the assay, cells were washed and incubated with XF Base Medium supplemented with $2 \mathrm{mM}$ L-glutamine, $1 \mathrm{mM}$ sodium pyruvate and $10 \mathrm{mM}$ glucose at $37^{\circ} \mathrm{C}$ without $\mathrm{CO}_{2}$ for $1 \mathrm{~h}$. Seahorse XFp Cell Mito Stress Test were performed with $1 \mu \mathrm{M}$ oligomycin, $1 \mu \mathrm{M}$ FCCP and $0.5 \mu \mathrm{M}$ Rotenone/antimycin A.

Mitochondrial analysis. To stain mitochondria, the cells were incubated with $500 \mathrm{nM}$ MitoTracker Red $\mathrm{CM}-\mathrm{H} 2 \mathrm{Xros}$ (Molecular Probes) in Full or $\Delta \mathrm{SG}$ medium for $30 \mathrm{~min}$ at $37^{\circ} \mathrm{C}$. Cell nuclei were counterstained with Hoechst33342. Images were obtained using BZ-X710 fluorescent microscopy (KEYENCE). All images in the individual panels were acquired under room temperature with the same settings. To quantify the amount of mitochondria and mitochondrial ROS, cells were seeded in 96-well round-bottom plates at a density of $5 \times 10^{5}$ cells/well and incubated with $500 \mathrm{nM}$ MitoTracker Red CM-H2Xros (Molecular Probes) or $5 \mu \mathrm{M}$ MitoSOX Red (Molecular Probes) in PBS with 1\% BSA and $2 \mathrm{mM}$ EDTA for $30 \mathrm{~min}$ at $37^{\circ} \mathrm{C}$ to stain mitochondria and to examine the amount of mitochondrial ROS, respectively. After washing, mean fluorescence intensity was measured using MACSQuant Analyzer 10 (Miltenyi Biotec) and the data was analyzed with FlowJo V10 (BD Biosciences).

Comprehensive metabolomic analysis.. Metabolomic analysis was performed at Human Metabolome Technologies (Tsuruoka, Japan). PeriMФs $\left(1.2 \times 10^{7}\right.$ cells) were seeded in $10 \mathrm{~cm}$ dish and cultured in DMEM medium containing 10\% FBS for $4 \mathrm{~h}$. Cells were then cultured in Full or $\Delta$ SG medium without FBS for $16 \mathrm{~h}$, followed by stimulation with control PBS or $100 \mathrm{ng} / \mathrm{ml}$ LPS for $6 \mathrm{~h}$. Cells were washed twice with $5 \%$ mannitol solution. Cellular metabolites were then extracted with $800 \mu \mathrm{l}$ of methanol and $500 \mu \mathrm{l}$ of distilled water containing HMT internal standard solution, as indicated by the manufacturer's instruction. The extract was centrifuged at $2300 \times g$ for $5 \mathrm{~min}$ at $4{ }^{\circ} \mathrm{C}$ and the supernatant was filtrated using an ULTRAFREE MC-PLHCC $5 \mathrm{kDa}$-cutoff filter unit $(\mathrm{HMT})$ by centrifugation at $9100 \times \mathrm{g}$ for $35 \mathrm{~min}$ at $4{ }^{\circ} \mathrm{C}$. Three biological replicates per condition were prepared. Metabolomic analysis was performed using CE-TOF MS/QqQ MS, and the metabolite peaks were quantified and normalized to the viable cell number. 
Amino acid analysis. Measurement of amino acids analysis in BMDMs was performed as described ${ }^{39}$. BMDMs $\left(4 \times 10^{6}\right.$ cells) seeded in $6 \mathrm{~cm}$ dish were washed twice with PBS and collected with $250 \mu \mathrm{l}$ of $\mathrm{CH} 3 \mathrm{OH}$, followed by addition of $10 \mu \mathrm{l}$ of $0.5 \mathrm{mg} / \mathrm{ml} 2$-isopropylmalic acid (Sigma-Aldrich) and10 $\mu \mathrm{l}$ of UL-13C,15 $\mathrm{N}$-amino acid mixture (Taiyo Nissan, Tokyo, Japan) as internal standards dissolved in distilled water. The mixture was incubated for $30 \mathrm{~min}$ at $37^{\circ} \mathrm{C}$ in a shaking incubator and then centrifuged at $16,000 \times g$ for 3 min at 4 ${ }^{\circ} \mathrm{C}$. The supernatant was evaporated to dryness. As the first derivatizing agent, $40 \mu \mathrm{lof} 20 \mathrm{mg} / \mathrm{ml}$ methoxyamine hydrochloride (Sigma-Aldrich) dissolved in pyridine was added and incubated for $90 \mathrm{~min}$ at $30{ }^{\circ} \mathrm{C}$ in a shaking incubator. The second derivatizing agent, $20 \mu \mathrm{l}$ of N-methyl-N-trimethylsilyl-trifluoroacetamide (GL Science, Tokyo, Japan), was mixed and incubated for $30 \mathrm{~min}$ at $37^{\circ} \mathrm{C}$ in a shaking incubator. The mixture was then centrifuged at $16,000 \times g$ for $3 \mathrm{~min}$ at $4{ }^{\circ} \mathrm{C}$, and the resultant supernatant was transferred to a vial for gas chromatography/mass spectrometry (GC/MS) measurement. GC/MS was carried out using GCMS-TQ8040 (Shimadzu Co., Kyoto, Japan) with a DB-5 capillary column ( $30 \mathrm{~m} \times 0.25 \mathrm{~mm}$ i.d.; $1 \mu \mathrm{m}$ film thickness; Agilent J \& W Scientific, Folsom, CA, USA). The inlet temperature was set at $250^{\circ} \mathrm{C}$, and the injection volume was $1 \mu \mathrm{L}$ (splitless mode). The GC column temperature was programmed to remain at $100^{\circ} \mathrm{C}$ for $4 \mathrm{~min}$, followed by a $10{ }^{\circ} \mathrm{C} \mathrm{min}{ }^{-1}$ linear ramp to a final temperature of $320^{\circ} \mathrm{C}$, which was held for $11 \mathrm{~min}$. Helium was used as a carrier gas at a flow rate of $1.1 \mathrm{~mL} \mathrm{~min}{ }^{-1}$. The transfer line and ion source temperatures were maintained at $280{ }^{\circ} \mathrm{C}$ and $200{ }^{\circ} \mathrm{C}$, respectively. For ionization, the electron impact mode at $70 \mathrm{eV}$ was used. Argon gas was used as a collision-induced dissociation gas. Metabolites were detected using the Smart Metabolites Database (Shimadzu), which included the relevant multiple reaction monitoring (MRM) method file and data regarding the GC analytical conditions, MRM parameters, and retention index used for amino acid measurement following the report. The detected values were quantified and normalized to the viable cell number.

Quantification and statistical analysis. All experiments were done at least three times independently. Data were analyzed using Prism Version 6 software (GraphPad). For data with two groups, unpaired t-tests were performed if homogeneity of variance and normality were confirmed, and by Welch's test if not confirmed. The data were analyzed by one-way analysis of variance (ANOVA) followed by the post hoc Tukey-Kramer's multiple comparison test for the comparison among 3 or more groups. When the values were compared only with the control values, Dunnett's multiple comparison tests were used instead of Tukey-Kramer's test. The time dependent changes were analyzed by repeated measures analysis of variance (ANOVA) with Bonferroni correction for comparisons between multiple groups and unpaired t-test for comparisons between two groups. Differences were assessed with two-side test with an $\alpha$ level of 0.05 and 0.01 . Data are presented as the mean $\pm 95 \%$ Confidence Interval (CI). $p<0.05$ was considered statistically significant.

Study approval. All animal experiments were conducted in accordance with the guidelines for the care and use of laboratory animals of Nagoya University. The protocols were approved by the Animal Care and Use Committee, Research Institute of Environmental Medicine, Nagoya University (Approval Number 20253).

\section{Data availability}

The dataset generated in this publication have been deposited in NCBI's Gene Expression Omnibus and are accessible through GEO: GSE156325 (https://www.ncbi.nlm.nih.gov/geo/query/acc.cgi?acc=GSE156325). Further information and requests for resources and reagents should be directed to and will be fulfilled by the Lead Contact, Takayoshi Suganami (suganami@riem.nagoya-u.ac.jp) or Ayaka Ito (aito@riem.nagoya-u.ac.jp).

Received: 13 November 2020; Accepted: 6 May 2021

Published online: 27 May 2021

\section{References}

1. Wynn, T. A. \& Vannella, K. M. Macrophages in tissue repair, regeneration, and fibrosis. Immunity 44, 450-462. https://doi.org/10. 1016/j.immuni.2016.02.015 (2016).

2. O'Neill, L. A., Kishton, R. J. \& Rathmell, J. A guide to immunometabolism for immunologists. Nat. Rev. Immunol. 16, 553-565. https://doi.org/10.1038/nri.2016.70 (2016).

3. O’Neill, L. A. \& Pearce, E. J. Immunometabolism governs dendritic cell and macrophage function. J. Exp. Med. 213, 15-23. https:// doi.org/10.1084/jem.20151570 (2016).

4. Iwasaki, Y. et al. Activating transcription factor 4 links metabolic stress to interleukin-6 expression in macrophages. Diabetes 63 , 152-161. https://doi.org/10.2337/db13-0757 (2014).

5. Suganami, T. et al. Role of the Toll-like receptor 4/NF-kappaB pathway in saturated fatty acid-induced inflammatory changes in the interaction between adipocytes and macrophages. Arterioscler. Thromb. Vasc. Biol. 27, 84-91. https://doi.org/10.1161/01.ATV. 0000251608.09329.9a (2007).

6. Shi, H. et al. TLR4 links innate immunity and fatty acid-induced insulin resistance. J. Clin. Investig. 116, 3015-3025. https://doi. org/10.1172/jci28898 (2006).

7. Miyajima, M. Amino acids: Key sources for immunometabolites and immunotransmitters. Int. Immunol. 32, 435-446. https://doi. org/10.1093/intimm/dxaa019 (2020).

8. Yang, M. \& Vousden, K. H. Serine and one-carbon metabolism in cancer. Nat. Rev. Cancer 16, 650-662. https://doi.org/10.1038/ nrc.2016.81 (2016).

9. Ma, E. H. et al. Serine is an essential metabolite for effector T cell expansion. Cell Metab. 25, 345-357. https://doi.org/10.1016/j. cmet.2016.12.011 (2017).

10. Ron-Harel, N. et al. Mitochondrial biogenesis and proteome remodeling promote one-carbon metabolism for T cell activation. Cell Metab. 24, 104-117. https://doi.org/10.1016/j.cmet.2016.06.007 (2016).

11. Possemato, R. et al. Functional genomics reveal that the serine synthesis pathway is essential in breast cancer. Nature 476, 346-350. https://doi.org/10.1038/nature10350 (2011). 
12. Labuschagne, C. F., Van Den Broek, N. J., Mackay, G. M., Vousden, K. H. \& Maddocks, O. D. Serine, but not glycine, supports onecarbon metabolism and proliferation of cancer cells. Cell Rep. 7, 1248-1258. https://doi.org/10.1016/j.celrep.2014.04.045 (2014).

13. Maddocks, O. D. K. et al. Corrigendum: Modulating the therapeutic response of tumours to dietary serine and glycine starvation. Nature 548, 122. https://doi.org/10.1038/nature23471 (2017).

14. Sakai, K., Shimizu, H., Koike, T., Furuya, S. \& Watanabe, M. Neutral amino acid transporter ASCT1 is preferentially expressed in L-Ser-synthetic/storing glial cells in the mouse brain with transient expression in developing capillaries. J. Neurosci. 23, 550-560 (2003).

15. Zhou, X. et al. Serine prevents LPS-induced intestinal inflammation and barrier damage via p53-dependent glutathione synthesis and AMPK activation. J. Funct. Foods 39, 225-232. https://doi.org/10.1016/j.jff.2017.10.026 (2017).

16. Zhai, P. P. et al. Reduction of inflammatory responses by L-serine treatment leads to neuroprotection in mice after traumatic brain injury. Neuropharmacology 95, 1-11. https://doi.org/10.1016/j.neuropharm.2015.02.026 (2015).

17. Zhou, X., Zhang, Y., Wu, X., Wan, D. \& Yin, Y. Effects of dietary serine supplementation on intestinal integrity, inflammation and oxidative status in early-weaned piglets. Cell Physiol. Biochem. 48, 993-1002. https://doi.org/10.1159/000491967 (2018).

18. He, F. et al. L-serine lowers the inflammatory responses during Pasteurella multocida infection. Infect Immun. https://doi.org/10. 1128/IAI.00677-19 (2019).

19. Rodriguez, A. E. et al. Serine metabolism supports macrophage IL-1 $\beta$ production. Cell Metab. 29, 1-9. https://doi.org/10.1016/j. cmet.2019.01.014 (2019).

20. Yu, W. et al. One-carbon metabolism supports S-adenosylmethionine and histone methylation to drive inflammatory macrophages. Mol. Cell 75, 1-14. https://doi.org/10.1016/j.molcel.2019.06.039 (2019).

21. Wang, Y. et al. Rotavirus infection alters peripheral T-cell homeostasis in children with acute diarrhea. J. Virol. 81, 3904-3912. https://doi.org/10.1128/JVI.01887-06 (2007).

22. Yarilina, A., Park-Min, K. H., Antoniv, T., Hu, X. \& Ivashkiv, L. B. TNF activates an IRF1-dependent autocrine loop leading to sustained expression of chemokines and STAT1-dependent type I interferon-response genes. Nat. Immunol. 9, 378-387. https:// doi.org/10.1038/ni1576 (2008).

23. Ravez, S., Spillier, Q., Marteau, R., Feron, O. \& Frédérick, R. Challenges and opportunities in the development of serine synthetic pathway inhibitors for cancer therapy. J. Med. Chem. 60, 1227-1237. https://doi.org/10.1021/acs.jmedchem.6b01167 (2017).

24. Gosset, P., Wallaert, B., Tonnel, A. B. \& Fourneau, C. Thiol regulation of the production of TNF-alpha, IL-6 and IL-8 by human alveolar macrophages. Eur. Respir. J. 14, 98-105. https://doi.org/10.1034/j.1399-3003.1999.14a17.x (1999).

25. Eming, S. A., Wynn, T. A. \& Martin, P. Inflammation and metabolism in tissue repair and regeneration. Science 356, $1026-1030$. https://doi.org/10.1126/science.aam7928 (2017).

26. Chaneton, B. et al. Serine is a natural ligand and allosteric activator of pyruvate kinase M2. Nature 491, 458-462. https://doi.org/ 10.1038/nature11540 (2012).

27. Sena, L. A. \& Chandel, N. S. Physiological roles of mitochondrial reactive oxygen species. Mol. Cell 48, 158-167. https://doi.org/ 10.1016/j.molcel.2012.09.025 (2012).

28. Ganeshan, K. \& Chawla, A. Metabolic regulation of immune responses. Annu. Rev. Immunol. 32, 609-634. https://doi.org/10.1146/ annurev-immunol-032713-120236 (2014).

29. Márquez, S. et al. Tricarboxylic acid cycle activity and remodeling of glycerophosphocholine lipids support cytokine induction in response to fungal patterns. Cell Rep. 27, 525-536. https://doi.org/10.1016/j.celrep.2019.03.033 (2019).

30. Palsson-McDermott, E. M. et al. Pyruvate kinase M2 regulates Hif- $1 \alpha$ activity and IL-1 $\beta$ induction and is a critical determinant of the warburg effect in LPS-activated macrophages. Cell Metab. 21, 65-80. https://doi.org/10.1016/j.cmet.2014.12.005 (2015).

31. De Miranda, J., Panizzutti, R., Foltyn, V. N. \& Wolosker, H. Cofactors of serine racemase that physiologically stimulate the synthesis of the N-methyl-D-aspartate (NMDA) receptor coagonist D-serine. Proc. Natl. Acad. Sci. USA 99, 14542-14547. https://doi.org/ 10.1073/pnas.222421299 (2002).

32. Ohshima, K. et al. Serine racemase enhances growth of colorectal cancer by producing pyruvate from serine. Nat. Metab. 2, 81-96. https://doi.org/10.1038/s42255-019-0156-2 (2020).

33. Maddocks, O. D. et al. Serine starvation induces stress and p53-dependent metabolic remodelling in cancer cells. Nature 493, 542-546. https://doi.org/10.1038/nature11743 (2013).

34. Diehl, F. F., Lewis, C. A., Fiske, B. P. \& Vander Heiden, M. G. Cellular redox state constrains serine synthesis and nucleotide production to impact cell proliferation. Nat. Metab. 1, 861-867. https://doi.org/10.1038/s42255-019-0108-x (2019).

35. Ip, W. K. E., Hoshi, N., Shouval, D. S., Snapper, S. \& Medzhitov, R. Anti-inflammatory effect of IL-10 mediated by metabolic reprogramming of macrophages. Science 356, 513-519. https://doi.org/10.1126/science.aal3535 (2017).

36. Formentini, L. et al. Mitochondrial ROS production protects the intestine from inflammation through functional M2 macrophage polarization. Cell Rep. 19, 1202-1213. https://doi.org/10.1016/j.celrep.2017.04.036 (2017).

37. Mills, E. L. et al. Succinate dehydrogenase supports metabolic repurposing of mitochondria to drive inflammatory macrophages. Cell 167, 457-470. https://doi.org/10.1016/j.cell.2016.08.064 (2016).

38. Suganami, T., Nishida, J. \& Ogawa, Y. A paracrine loop between adipocytes and macrophages aggravates inflammatory changes: Role of free fatty acids and tumor necrosis factor alpha. Arterioscler. Thromb. Vasc. Biol. 25, 2062-2068. https://doi.org/10.1161/ 01.ATV.0000183883.72263.13 (2005).

39. Mizusawa, A. et al. BDK deficiency in cerebral cortex neurons causes neurological abnormalities and affects endurance capacity. Nutrients https://doi.org/10.3390/nu12082267 (2020).

\section{Acknowledgements}

We thank Dr. Norihiko Takeda (Jichi Medical University) and the members of the Suganami laboratory for their helpful discussions, and Center for Animal Research and Education (CARE), Nagoya University for support on animal experiments. This work was supported in part by Grants-in-Aid for Scientific Research from the Ministry of Education, Culture, Sports, Science and Technology of Japan (20H03447, 20H05503, and 20H04944 to T.S., 19 K11765 and 19KK0249 to A.I.) and Japan Agency for Medical Research and Development (CREST) (20gm1210009s0102 to T.S.). This study was also supported by research grants from Smoking Research Foundation (T.S.), Astellas Foundation for Research on Metabolic Disorders, Kowa Life Science Foundation (A.I.), The Hori Sciences and Arts Foundation, and Takeda Science Foundation (T.S., A.I.).

\section{Author contributions}

K.K. and H.O. designed and performed experiments and wrote the manuscript. I.S. performed transcriptomic and metabolomic analysis and wrote the manuscript. M.T. and Y.I. analyzed data and contributed to discussion. Y.K. performed metabolomic analysis. T.M. and H.S. generated essential materials. S.A. supervised statistical analysis. Y.O. and H.A. supervised the study. A.I. and T.S. conceptualized and supervised the study, acquired funding, and wrote and edited the manuscript. 


\section{Competing interests}

The authors declare no competing interests.

\section{Additional information}

Supplementary Information The online version contains supplementary material available at https://doi.org/ 10.1038/s41598-021-90086-w.

Correspondence and requests for materials should be addressed to A.I. or T.S.

Reprints and permissions information is available at www.nature.com/reprints.

Publisher's note Springer Nature remains neutral with regard to jurisdictional claims in published maps and institutional affiliations.

(c) (1) Open Access This article is licensed under a Creative Commons Attribution 4.0 International License, which permits use, sharing, adaptation, distribution and reproduction in any medium or format, as long as you give appropriate credit to the original author(s) and the source, provide a link to the Creative Commons licence, and indicate if changes were made. The images or other third party material in this article are included in the article's Creative Commons licence, unless indicated otherwise in a credit line to the material. If material is not included in the article's Creative Commons licence and your intended use is not permitted by statutory regulation or exceeds the permitted use, you will need to obtain permission directly from the copyright holder. To view a copy of this licence, visit http://creativecommons.org/licenses/by/4.0/.

(C) The Author(s) 2021 